\title{
Changes in the gut bacterial communities in colon cancer surgery patients: an observational study
}

\author{
Mohamed Abbas ${ }^{1 *}$ (D) Nadia Gaïa ${ }^{2}$, Nicolas C. Buchs ${ }^{3}$, Vaihere Delaune ${ }^{3}$, Myriam Girard², Diego O. Andrey ${ }^{4}$, \\ Jeremy Meyer ${ }^{3}$, Jacques Schrenzel ${ }^{2,4,5}$, Frédéric Ris $^{3}$, Stephan Harbarth ${ }^{1,4}$ and Vladimir Lazarevic ${ }^{2}$
}

\begin{abstract}
Background: Colon surgery has been shown to modulate the intestinal microbiota. Our objective was to characterize these changes using state-of-the-art next generation sequencing techniques.

Methods: We performed a single-centre prospective observational cohort study to evaluate the changes in the gut microbiota, i.e., taxon distribution, before and after elective oncologic colon surgery in adult patients with different antimicrobial prophylaxis regimens (standard prophylaxis with cefuroxime/metronidazole versus carbapenems for extended-spectrum beta-lactamase-producing Enterobacterales [ESBL-E] carriers). We obtained rectal samples on the day of surgery, intraoperative luminal samples, and rectal or stoma samples 3 days after surgery. We performed metataxonomic analysis based on sequencing of the bacterial 165 rRNA gene marker. Similarities and differences between bacterial communities were assessed using Bray-Curtis similarity, visualised using principal coordinates analysis and statistically tested by PERMANOVA. Comparison of taxa relative abundance was performed using ANCOM.

Results: We included 27 patients between March 27, 2019 and September 17, 2019. The median age was 63.6 years (IQR 56.4-76.3) and 44\% were females. Most (81\%) patients received standard perioperative prophylaxis as they were not ESBL carriers. There was no significant association between ESBL carriage and differences in gut microbiome. We observed large and significant increases in the genus Enterococcus between the preoperative/intraoperative samples and the postoperative sample, mainly driven by Enterococcus faecalis. There were significant differences in the postoperative microbiome between patients who received standard prophylaxis and carbapenems, specifically in the family Erysipelotrichaceae.
\end{abstract}

Conclusion: This hypothesis-generating study showed rapid changes in the rectal microbiota following colon cancer surgery.

Keywords: Gut microbiome, Colon cancer, Colon surgery, Antimicrobial prophylaxis

\section{Introduction}

Surgical site infections (SSI) are a leading cause of healthcare-associated infections [1]. Among surgical procedures, colorectal surgery is associated with the highest

\footnotetext{
*Correspondence: mohamed.abbas@hcuge.ch

${ }^{1}$ Infection Control Programme, Geneva University Hospitals and Faculty of Medicine, Geneva, Switzerland
}

Full list of author information is available at the end of the article incidence of SSI, despite widespread implementation of evidence-based preventive practices [2]. Indeed, in Switzerland, the average incidence of SSI in colon surgery is $14.4 \%$ [3].

Patient-level risk factors for SSI in colorectal surgery include age $[4,5]$, obesity [5-8], and diabetes [4]. Intraoperative risk factors include emergency surgery $[9,10]$, contaminated or dirty surgery $[5,10]$, duration of surgery, original author(s) and the source, provide a link to the Creative Commons licence, and indicate if changes were made. The images or other third party material in this article are included in the article's Creative Commons licence, unless indicated otherwise in a credit line to the material. If material is not included in the article's Creative Commons licence and your intended use is not permitted by statutory regulation or exceeds the permitted use, you will need to obtain permission directly from the copyright holder. To view a copy of this licence, visit http://creativecommons.org/licenses/by/4.0/. The Creative Commons Public Domain Dedication waiver (http://creativeco mmons.org/publicdomain/zero/1.0/) applies to the data made available in this article, unless otherwise stated in a credit line to the data. 
and creation of an ostomy [7, 9]; laparoscopic surgery is a protective factor $[5,11]$.

In the field of colorectal surgery, there is a body of indirect evidence suggesting that the microbiome plays an important role in the postoperative outcome, notably in the incidence of SSI, including anastomotic leak [12]. The current paradigm surrounding management of the high bacterial load prior to surgery is to maximise decontamination, either by perioperative antibiotic prophylaxis alone (one of the most effective measures) or in combination with bowel cleansing (mechanical bowel preparation) and/or topical oral antibiotics [13]. However, this decontamination indiscriminately affects both beneficial and potentially pathogenic bacteria.

There is a real gap in the literature regarding adequate description of the modulation in the intestinal microbiota in patients undergoing colon surgery using state-ofthe-art next-generation sequencing techniques.

Therefore, we performed a prospective observational study to evaluate changes in the gut microbiota in adult patients undergoing perioperative antibiotic prophylaxis regimens and elective colon surgery, and to assess differences between preoperative (rectal) and intraoperative (luminal) microbiome. Because all patients received perioperative antibiotic prophylaxis, we believe that the changes in the gut microbiota would be due to the impact of antibiotics.

\section{Methods}

We performed a single-centre prospective observational cohort study including adult patients who underwent elective colon surgery at Geneva University Hospitals, Switzerland, which is a regional tertiary centre.

Eligible patients were identified from the operating programme and were included if they were adults (age $\geq 18$ years), and were scheduled to undergo elective colon surgery for oncological reasons. Informed consent was obtained during the preoperative surgical visit. Included patients have also been enrolled in a prospective cohort in a national surveillance system of SSI (Swissnoso) [3]. Exclusion criteria were emergency colon surgery, rectal surgery, receiving any topical oral or systemic oral/parenteral antibiotic within 30 days preceding the colon surgery, and necessity of mechanical bowel preparation. Patients who were unable or unwilling to provide informed consent or for whom 30-day follow-up was difficult/impossible (e.g., residing overseas) were also excluded.

We collected patient data on their digestive colonisation status with extended-spectrum beta-lactamase producing Enterobacterales (ESBL-E) as well as demographic and medical data, from the electronic medical health record.
According to institutional perioperative prophylaxis guidelines, patients receive a second-generation cephalosporin (cefuroxime) plus metronidazole within $60 \mathrm{~min}$ preceding the incision, except those known to have intestinal colonisation with ESBL-E who receive ertapenem, as non-targeted prophylaxis is associated with an increased risk of SSI [14].

Patients in our study were all included in our institutional Fast-track protocol [15]. Briefly, nutritional status was assessed one month prior to surgery. In case of impaired nutritional status (as testified by low serum prealbumin levels and a Nutritional Risk Score (NRS) $\geq 3$ [16], patients received protein-enriched oral supplements. No dietary restrictions were enforced before surgery. Non-diabetic patients were given a hyperglycaemic oral solution (ProvideXtra ${ }^{\circledR}$, Fresenius Kabi, Germany) the night before $(400 \mathrm{ml})$ and two hours $(200 \mathrm{ml})$ prior to surgery. A low-fibre diet was introduced at postoperative day 1 , and was continued for approximately two weeks. Protein-enriched oral supplements were given between postoperative days 2 and 7 .

\section{Sample collection}

Three samples were collected from included patients on two occasions using Copan ESwab (480 CFA, Regular Flocked Swab with Liquid Amies Medium). The first sample was a rectal swab collected on the day of surgery, just after the patient had undergone general anaesthesia. The second was a swab of luminal stool in the proximal colonic divided segment. The third sample was a rectal (or stoma) swab performed on postoperative day 3 . The samples were immediately frozen at $-80{ }^{\circ} \mathrm{C}$, pending batched metataxonomic analysis.

\section{DNA extraction, 16S rRNA gene amplification and sequencing}

Thawed ESwab was homogenised by vortexing during $1 \mathrm{~min}$. DNA was extracted from $250 \mu \mathrm{L}$ of suspension using ZymoBIOMICS DNA Miniprep kit (Zymo Research, US) and eluted in $60 \mu \mathrm{L}$ of $\mathrm{H}_{2} \mathrm{O}$. Purified DNA was quantified using the Qubit dsDNA BR Assay Kit (Thermo Fisher Scientific, US) according to manufacturer's instructions and stored at $-20^{\circ} \mathrm{C}$. Three no-sample controls were performed by extracting DNA using the same extraction procedure but omitting the addition of a sample.

The V3-4 region of the bacterial 16S rRNA genes was amplified using $1 \mathrm{ng}$ of extracted DNA or $6 \mu \mathrm{L}$ of the extract of no-sample controls, as previously described [17].

The sequencing library construction using the MetaFast protocol, Illumina MiSeq $2 \times 300$ sequencing with MiSeq Reagent Kit v3 and initial sequence processing 
(demultiplexing and removal of adapter and primer sequences) were performed externally at Fasteris (Planles-Ouates, Switzerland).

\section{Bioinformatics}

Paired reads were quality filtered and joined using PEAR v0.9.11 (-m 470 -n 390 -t 150 -v 10 -q 33 -p 0.0001 -u 0) [18]. Merged sequence reads were clustered into zeroradius operational taxonomic units (zOTUs) using UNOISE3 [19] from the USEARCH v11.0.667 package [20].

From the sample dataset, we removed zOTUs matching any of the following criteria: (i) presented $<90 \%$ identity to reference bacterial sequences in the EzBioCloud $16 \mathrm{~S}$ database [21] (downloaded on the $19^{\text {th }}$ of August 2019) as revealed by USEARCH [22] (-id 0.90 -query_cov 0.99); (ii) were represented by $\leq 10$ counts; and (iii) had higher relative abundance in no-sample controls than in clinical samples. zOTUs were classified using EzBioCloud 16S database via MOTHUR v1.43.0 [23] using command classify.seqs (method $=$ wang cutoff $=80$ ). Sequencing data were submitted to the European Nucleotide Archive (ENA; www.ebi.ac.uk/ena; study number: PRJEB44214).

\section{Bacterial community comparisons}

Bacterial communities were clustered using Bray-Curtis similarity [24] matrix constructed in PRIMER v7 (PRIMER-E Ltd, Plymouth, UK) based on square roottransformed relative abundance of zOTUs. Similarities and differences between communities were visualised using principal coordinates analysis ( $\mathrm{PCoA})$. To assess significance of differences in overall microbiota taxonomic composition between groups defined by categorical variables, we used permutational multivariate analysis of variance [25] (PERMANOVA) test (adonis2 function in vegan v2.5-7 $R$ v3.6.1 package) with 9,999 permutations. The homogeneity of multivariate dispersion (average distance to the group centroid) was assessed by PERMDISP [26] using betadisper and permutest (with 9,999 permutations) functions in vegan [27]. To analyse the relationship between bacterial community profiles and quantitative variables, we used a distance-based linear model (DISTLM, PRIMER) with 9,999 permutations.

To identify differentially abundant taxa (from phylum down to the species level), we used the analysis of composition of microbiomes (ANCOM) [28] with the following settings: adjusted $=F$, repeated $=F$, multcorr $=2$ ("less stringent" multiple comparison correction), sig $=0.05$, prev.cut $=0.75$ (features not observed in $\geq 75 \%$ samples were omitted) and, when appropriate, paired.test ancom $=$ paired. The results that passed the 0.6 threshold were considered significant.
The Shannon diversity index was calculated from the relative abundance of zOTUs in PRIMER.

\section{Ethical approval}

The study was approved by the local Ethics Committee (Geneva, no. 2018-02379) and complied with the declaration of Helsinki.

\section{Results}

Between March 27, 2019 and September 17, 201928 adult patients were scheduled to undergo elective colorectal cancer surgery and were therefore considered as eligible for the study. One patient was excluded because the postoperative sample was not collected, leaving 27 patients in the final analysis. The median age was 63.6 years and $44 \%$ were female. One patient was operated for a non-resectable polyp, two were operated for neuroendocrine tumours, and the remaining 24 patients had adenocarcinoma. Table 1 summarises other patient characteristics.

Most (81.5\%) patients received standard perioperative antimicrobial prophylaxis as they were not ESBL-E carriers (Table 2). Four patients (14.8\%) received ertapenem due to ESBL-E carriage, although 1 patient was subsequently found to have a negative screening swab. One patient received meropenem due to a history of carriage of carbapenem resistant Enterobacterales (Escherichia coli harbouring the OXA-48 gene, phenotypically susceptible to meropenem). Two patients developed SSI: one patient who received ertapenem for ESBL E. coli carriage developed superficial SSI with Streptococcus anginosus, Staphylococcus lugdunensis, and Enterococcus faecalis, as revealed by routine culture; another patient, who received standard prophylaxis, developed organ/space SSI and the recovered intraoperative microorganisms were S. anginosus, E. faecalis, and Colinsella aerofaciens, as revealed by routine culture.

\section{Differences in bacterial communities among patients and sample types}

There were significant differences of bacterial taxonomic profiles in the analysis with PERMANOVA: postoperative vs preoperative rectal swabs in non-stoma patients $\left(P=0.005, R^{2}=0.0435, F=1.9121\right.$; only patients with standard prophylaxis: $\mathrm{P}=0.0056, \mathrm{R}^{2}=0.0626$, $\mathrm{F}=2.1372$ ); preoperative rectal vs intraoperative luminal swabs $\left(\mathrm{P}=0.0145, \mathrm{R}^{2}=0.0317, \mathrm{~F}=1.7046\right)$; and postoperative rectal vs intraoperative luminal swabs of non-stoma patients $\left(\mathrm{P}=0.0001, \mathrm{R}^{2}=0.0948, \mathrm{~F}=4.398\right.$; only patients with standard prophylaxis: $\mathrm{P}=0.0001$, $\mathrm{R}^{2}=0.1162, \mathrm{~F}=4.2071$ ).

In terms of changes in individual bacterial taxa, we observed large and significant increases in the genus 
Table 1 Characteristics of patients

\begin{tabular}{|c|c|}
\hline Characteristic & $\begin{array}{l}\text { Frequency } \\
\text { (\%) or median } \\
\text { (IQR) }\end{array}$ \\
\hline Age (years) & $63.6(56.4-76.3)$ \\
\hline Gender (female) & $12(44.4)$ \\
\hline Body mass index $\left(\mathrm{kg} / \mathrm{m}^{2}\right)$ & $25.0(22.6-27.3)$ \\
\hline Prealbumin ${ }^{\mathrm{a}}(\mathrm{mg} / \mathrm{l})$ & $270(232-306)$ \\
\hline Preoperative blood glucose (mmol/l) & $5.7(5.3-6.9)$ \\
\hline Preoperative haemoglobin (g/l) & $133(113-145)$ \\
\hline Postoperative haemoglobin, day 3 (g/l) & $118(105-122)$ \\
\hline \multicolumn{2}{|l|}{ MDR-GNB intestinal carriage ${ }^{b}$} \\
\hline None & $20(74.7)$ \\
\hline ESBL & $3(11.1)$ \\
\hline CRE and ESBL & $1(3.7)$ \\
\hline \multicolumn{2}{|l|}{ Type of tumour } \\
\hline Adenocarcinoma & $24(88.9)$ \\
\hline Neuroendocrine tumour & $2(7.4)$ \\
\hline Non-resectable polyp & $1(3.7)$ \\
\hline \multicolumn{2}{|l|}{ Tumour location } \\
\hline Ascending colon & $12(44.4)$ \\
\hline Transverse colon & $2(7.4)$ \\
\hline Descending colon & $7(25.9)$ \\
\hline Sigmoid colon & $6(22.2)$ \\
\hline \multicolumn{2}{|l|}{ TNM stage ${ }^{* c}$} \\
\hline $\mathrm{T} 1$ & $2(7.4)$ \\
\hline $\mathrm{T} 2$ & $6(22.2)$ \\
\hline T3 & $9(33.3)$ \\
\hline T4 & $6(22.2)$ \\
\hline
\end{tabular}

$C R E$ carbapenem-resistant Enterobacteriaceae, ESBL extended-spectrum betalactamase, IQR interquartile range, MDR-GNB multi-drug resistant Gram-negative bacteria

* for adenocarcinoma patients only $(n=24)$

a 1 missing value

b 3 missing values

c 1 missing value

Enterococcus and species E. faecalis when preoperative rectal and intraoperative luminal swabs were compared to postoperative rectal samples (Additional file 1: Table S1). The abundance of unclassified Blautia and Agathobacter rectalis, both belonging to Lachnospiraceae, decreased in postoperative samples. These changes were significant when all patients were considered together or when we analysed only those that received standard prophylaxis. In patients under carbapenem prophylaxis we observed similar changes but with no statistical significance, likely due to the small sample size $(n=5)$. For example, the median relative abundance of Enterococcus in postoperative samples was even higher in patients with carbapenem- than in those with standard prophylaxis (10.3 vs $1.3 \%$ ).
Table 2 Operative characteristics

\begin{tabular}{|c|c|}
\hline Characteristic & $\begin{array}{l}\text { Frequency } \\
\text { (\%) or median } \\
\text { (IQR) }\end{array}$ \\
\hline \multicolumn{2}{|c|}{ Perioperative antimicrobial prophylaxis } \\
\hline Cefuroxime + metronidazole & $22(81.5)$ \\
\hline Ertapenem & $4(14.8)$ \\
\hline Meropenem* & $1(3.7)$ \\
\hline \multicolumn{2}{|l|}{ Laparoscopically-assisted surgery } \\
\hline Yes & $21(77.8)$ \\
\hline No & $3(11.1)$ \\
\hline Conversion to laparotomy & $3(11.1)$ \\
\hline Duration of procedure (minutes) & $190(163-243)$ \\
\hline Restoration of bowel continuity & $22(81.5)$ \\
\hline Intraoperative complication & $1(3.7)$ \\
\hline \multicolumn{2}{|l|}{ Surgical site infection } \\
\hline Superficial & $1(3.7)$ \\
\hline Organ/space & $1(3.7)$ \\
\hline
\end{tabular}

In patients receiving standard prophylaxis, several taxa had higher levels in rectal swabs than in luminal samples. These included anaerobic gram-negative short rod-shaped Porphyromonas (phylum Bacteroidetes) and anaerobic gram-positive cocci from the Peptoniphilaceae family (Additional file 1: Table S1). Peptoniphilaceae was the only representative of the order Tissierellales (Fig. 1) and the class Tissierellia (phylum Firmicutes) and was notably represented by the genera Peptoniphilus, Finegoldia, Anaerococcus, Murdochiella, Fenollaria and Ezakiella.

In patients receiving standard prophylaxis (all of the 5 stoma patients and 17/22 non-stoma patients), stoma and rectal swab microbiota were significantly different $\left(P=0.0032, R^{2}=0.0862, F=1.8865\right)$. Compared to rectal swabs, stoma samples had significantly higher proportion of the order Enterobacterales (Fig. 1) and associated higher-level taxa (class Gammaproteobacteria and phylum Proteobacteria). In addition, the diversity of the stomal microbiota was significantly lower to that or rectal and luminal swabs (Fig. 2) and lower within-patient similarity to preoperative swabs as compared to postoperative rectal swabs (Fig. 3).

Despite all above mentioned variations (Fig. 4A), the samples also clustered by individual (all patients, $\mathrm{P}=0.0096, \mathrm{R}^{2}=0.02257, \mathrm{~F}=1.8241$; non-stoma patients $\left.\mathrm{P}=0.0001, \mathrm{R}^{2}=0.07643, \mathrm{~F}=2.6068\right)($ Fig. $4 \mathrm{~B})$.

\section{Microbiota differences related to the prophylaxis type}

Differences in the postoperative rectal swab microbiota between patients with standard prophylaxis and those 


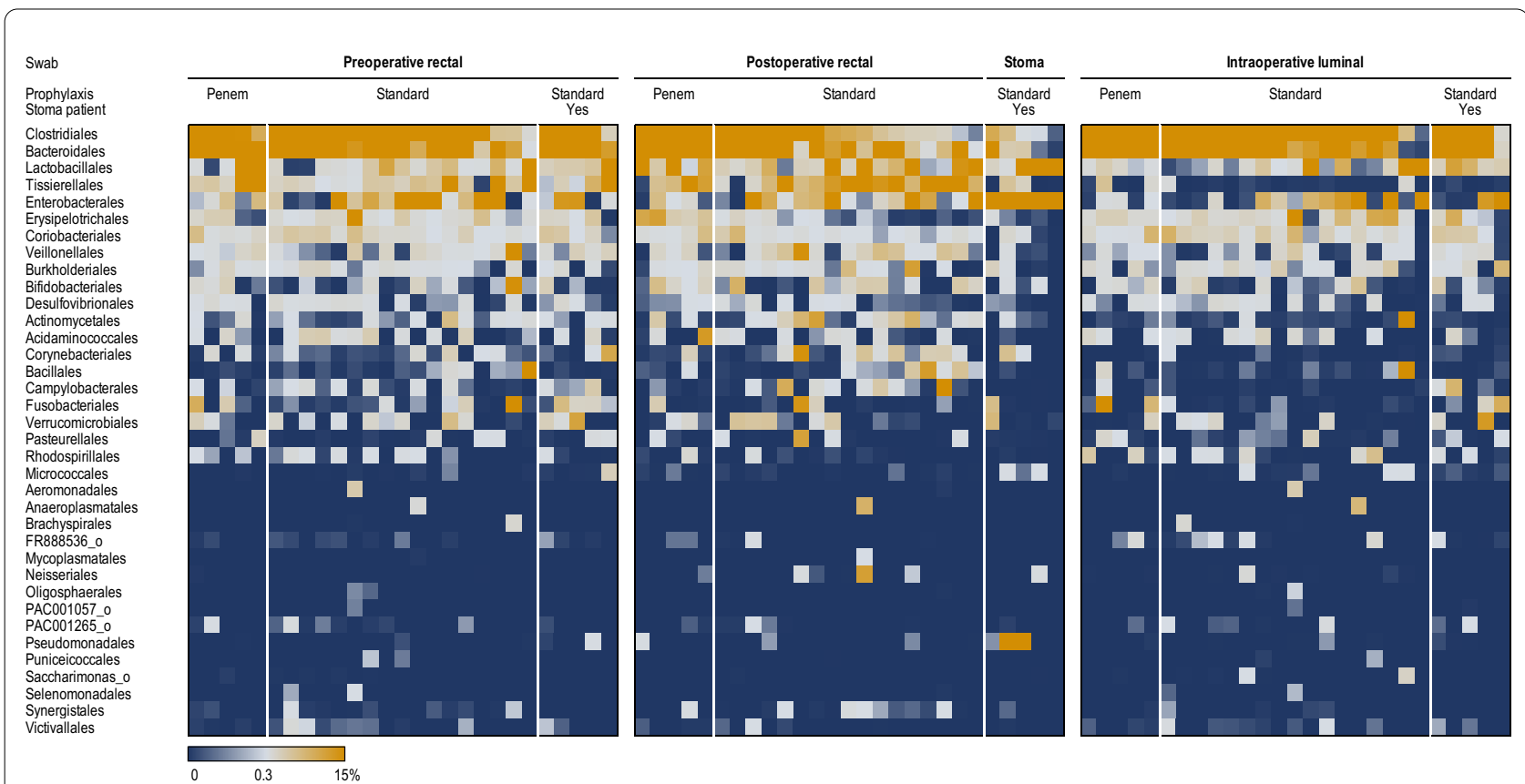

Fig. 1 Heat map of the relative abundance of bacterial orders across different sample types and sampling points. The columns correspond to the patients, ordered by decreasing relative abundance of Clostridiales within each group

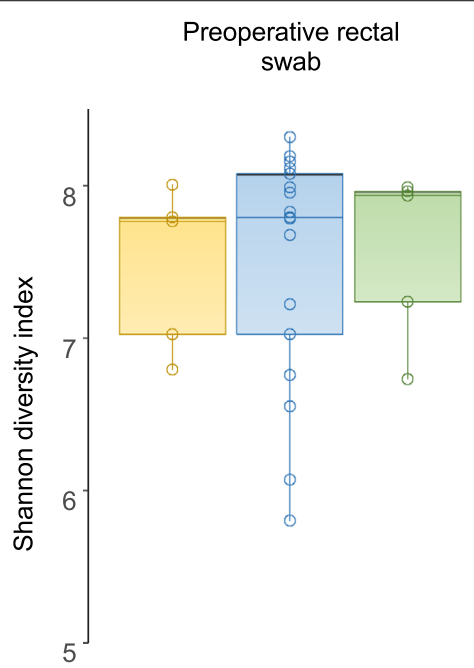

$\begin{array}{cc}\begin{array}{c}\text { Postoperative } \\ \text { rectal swab }\end{array} & \text { Stoma } \\ \text { swab }\end{array}$
swab

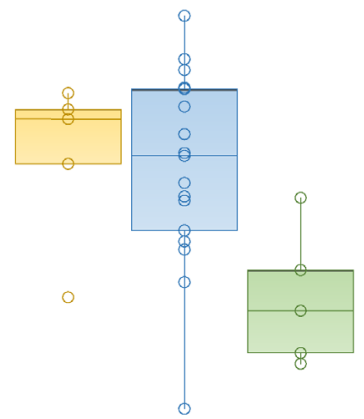

Intraoperative luminal swab

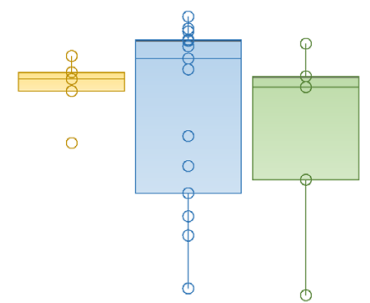

Penem prophylaxis

Standard prophylaxis

Stoma patients with standard prophylaxis

Fig. 2 Bacterial diversity across samples from different sites and sampling points. Differences between stoma swabs and all presented groups of luminal or rectal swabs were statistically significant $(P<0.05$ by Wilcoxon rank sum test)

with carbapenem administration were significant (PERMANOVA $P=0.03, R^{2}=0.0711, F=1.5301$ ), which was not the case when corresponding preoperative swabs were analysed $(\mathrm{P}=0.4338)$. The difference in multivariate dispersion of microbial communities (Fig. 4A) between the two treatments were close to the significance threshold (PERMDISP $\mathrm{P}=0.0557$ ) for postoperative but not for the corresponding preoperative samples (PERMDISP $\mathrm{P}=0.5629$ ).

Postoperative rectal swabs of patients who received standard prophylaxis had significantly higher relative abundances of the class Erysipelotrichii and lowerlevel taxa belonging to it (order Erysipelotrichales, family Erysipelotrichaceae, genus Longicatena and a 


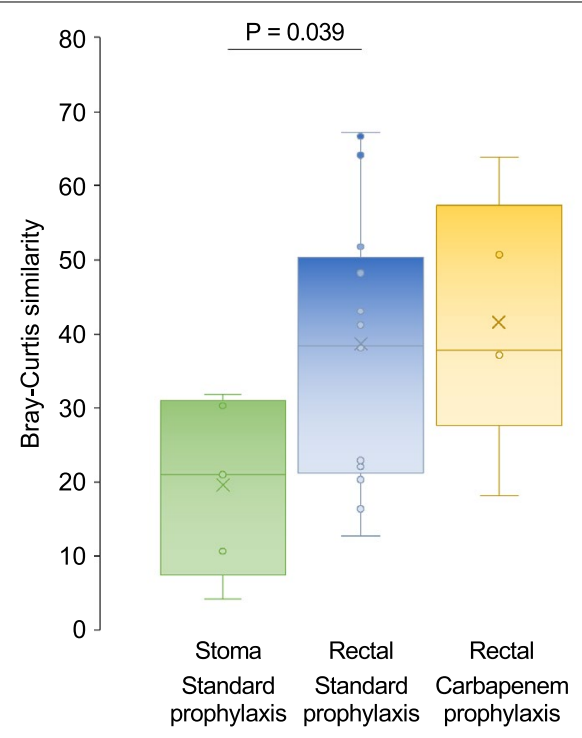

Fig. 3 Bray Curtis similarity between preoperative (rectal) and postoperative (stoma or rectal) samples in patients who received cefuroxime + metronidazole (standard) versus carbapenem prophylaxis. $P$ value is indicated only when significant $(P<0.05$ by Wilcoxon rank sum test)

not-yet-validated Longicatena species JH590969_s) when compared to those with carbapenem, as revealed by ANCOM test.

\section{Associations between microbiota and patient characteristics}

The relationship between patient characteristics and differences in gut microbiome are shown in Table 3.
Briefly, although there was a trend towards an association between body mass index and differences in the preoperative gut microbiome, these were not significant.

There were no significant associations between tumour characteristics and changes in the gut microbiome, except for location of tumour and intraoperative microbiome (Table 3, Additional file 2: Supplementary Fig. 1), which possibly reflects variations in microbiota along the longitudinal gut axis [29]. The microbiota from the distal colon (descending and sigmoid segments considered together), when compared to those from the proximal (ascending plus transverse) part, were significantly depleted in Aerococcaceae/Granulicatella [median $0.0019, \mathrm{IQR}(0.0011-0.0036)$ vs $0.0356(0.004-0.2765)]$ and Veillonella [0.0032 (0.0007-0.0207) vs $0.0559(0.018-$ 0.3472)], while being enriched in Christensenellaceae [(1.4842 (0.6272-2.3287) vs $0.0987(0.0016-0.2384)]$.

There was no significant association between E-ESBL carriage and differences in gut microbiome in any of the samples.

\section{Discussion}

This prospective hypothesis-generating pilot study evaluated changes in the rectal microbiota of colon cancer patients requiring a surgical intervention. The results suggest that surgery, including perioperative antimicrobial prophylaxis, induces rapid changes in the gut microbiota.

There is a dearth of studies evaluating perioperative changes in the rectal microbiome of colon cancer surgery patients. A prospective study on 54 US patients showed that both preoperative and postoperative composition
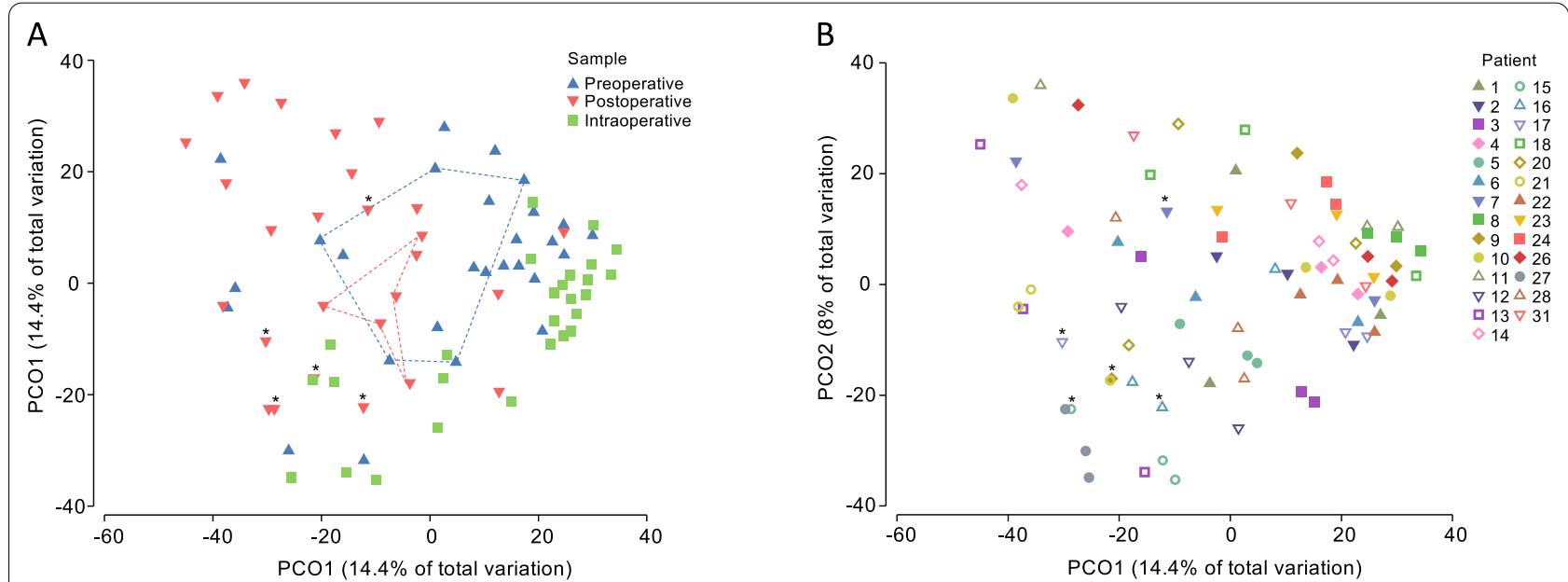

Fig. 4 PCoA of beta-diversity of bacterial communities. Each data point on the chart represents a patient-sample combination. A Differences between preoperative rectal swabs, intraoperative luminal swab and postoperative (day 3) rectal or stoma (asterisk) swabs. The five pre- or postoperative samples from the subjects who received carbapenem prophylaxis are connected by dashed lines. Other patients (including all stoma patients) received standard prophylaxis (cefuroxime + metronidazole). B Differences between patients 
Table 3 Associations between patient and tumour characteristics and overall bacterial profiles of preoperative rectal and intraoperative luminal gut microbiota

\begin{tabular}{lll}
\hline Characteristic & \multicolumn{2}{l}{ P value* } \\
\cline { 2 - 3 } & $\begin{array}{l}\text { Preoperative } \\
\text { rectal }\end{array}$ & Intraoperative luminal \\
\hline Age & 0.1571 & 0.1869 \\
Female gender & 0.9038 & 0.3199 \\
Body mass index & 0.0798 & 0.1681 \\
Prealbumin & 0.4161 & 0.5159 \\
Glucose & 0.6901 & 0.3293 \\
Preoperative haemoglobin & 0.2852 & 0.1709 \\
Postoperative haemoglobin & 0.5422 & 0.2148 \\
E-ESBL carriage & 0.6326 & 0.7367 \\
Tumour characteristics & & \\
Tumour location & 0.2698 & 0.0109 \\
(ascending/transverse vs & & (R2=0.03425 \\
descending/sigmoid) & & $\mathrm{F}=1.7623)$ \\
Tumour stage & 0.9298 & 0.9778 \\
(T1/T2 vs T3/T4) & & 0.2722 \\
Tumour budding & 0.1342 & \\
(BD1 vs BD2/BD3) & &
\end{tabular}

* based on PERMANOVA or DISTLM tests

of the rectal microbiome, in particular higher proportions of Proteobacteria and Bacteroidetes and lower levels of Actinobacteria and Firmicutes, were associated with postoperative ileus, but not SSI [30]. These results are not directly comparable to ours for several reasons: first, Shogan et al. [12] included both colon and rectal surgery patients. Second, they included patients with diverse surgical indications: $61 \%$ were operated for non-cancer related diseases such as inflammatory bowel disease, which may be associated both with altered rectal microbiome and development of postoperative complications. This is reflected also by the fact that $14 \%$ patients experienced postoperative ileus. Third, $82 \%$ of their patients underwent mechanical bowel preparation, which is also known to alter the microbiome. In contrast, our study was a more homogenous population, with minimal disruption of the microbiome apart from surgery and perioperative antimicrobial prophylaxis.

Van Praagh et al. published a cohort study evaluating the association between the local mucosal tissue microbiome at the site of anastomosis with the risk of anastomotic leakage of 123 colorectal surgery patients in the Netherlands, most of whom (95\%) were operated for cancer [31]. Anastomotic leakage was associated with increased relative abundance of Lachnospiraceae and Bacteroidaceae. Again, patients in their cohort underwent mechanical bowel preparation [32], and thus these results do not reflect the "native" microbiome.
The study by Jin et al. assessed the ability of the intraoperative tissue microbiome composition to predict postoperative ileus in colorectal cancer patients in China [33]. Their results suggest decreased relative abundance of Faecalibacterium in patients who would later develop postoperative ileus, consistent with the results by Shogan et al. [30].

Another observational study based on targeted PCR assays to evaluate changes in bacterial counts before and after colon surgery, showed decreases in beneficial obligate anaerobes and increases in pathogenic bacteria; this study did not have the potential to provide a comprehensive overview of the microbiome because it did not use next-generation sequencing-based techniques [34].

Several interventional human studies suggest that preoperative administration of probiotics or synbiotics to colorectal surgery patients may be associated with postoperative complications, including infection; these studies, however, are fraught with methodological shortcomings, and the level of uncertainty on the effectiveness of this intervention [35]. One study using $16 \mathrm{~S}$ rRNA gene-based metataxonomics was a single-centre randomized clinical trial which showed that perioperative administration of probiotics (Bifidobacterium longum) in patients undergoing colorectal surgery increases the proportions of Actinobacteria; there were, however, no statistically significant differences between the intervention group and control group either in terms of composition of the microbiota nor in terms of clinical outcome, probably due to the small size of the study [36].

Animal models show that colon surgery and perioperative measures (perioperative antibiotic prophylaxis, fasting, mechanical bowel preparation) alter the microbiome [12]. In rats, colectomy is associated with important increases in the amount of pathogenic bacteria (Enterococcus, Escherichia) [37].

Strengths of our study include state-of-the-art nextgeneration sequencing and bioinformatics techniques, a well-defined homogenous study population, as well as robust statistical analyses. Ours was a prospective study, decreasing the risk of bias associated with retrospective studies.

The main limitation of this study was the sample size, which was small, as was the number of events we had initially planned to evaluate (SSI). Despite this, we present interesting and novel findings, which will need to be confirmed in larger studies. Another limitation is the short duration of follow-up of the rectal/gut microbiome; indeed, it would have been interesting to study the dynamics of recovery of the different taxa, and investigate factors associated with this recovery. Finally, we only focused on the intestinal microbiome, whereas a more comprehensive approach also including the skin and 
nares microbiome should be adopted since the members of these communities can cause SSI [38, 39].

Understanding changes in the gut microbiome of colon cancer surgery patients is important, yet understudied [40]. Further, well-designed prospective studies with homogenous populations should be performed, in combination with other explanatory factors in order to obtain significant insights [41]. The research agenda should also include the changes of the gut microbiome specifically associated with mechanical bowel preparation and, more importantly, non-absorbable oral antimicrobials [42].

\section{Conclusions}

In conclusion, our results showed rapid changes in the rectal microbiota following colon surgery. We also observed differences in microbiota structure between stoma and rectal swabs, whereas previous studies showed good similarity between rectal swab and stool microbiota $[43,44]$. Our findings highlight that results obtained by using rectal swab (and possibly faecal matter) microbiota, as a proxy for colonic microbiota, should be interpreted with caution due to the differences between rectal and luminal colonic bacterial communities, notably in the relative abundance of anaerobic gram-positive cocci and gram-negative rods.

\section{Availability of data}

Sequencing data were submitted to the European Nucleotide Archive (ENA; www.ebi.ac.uk/ena; study number: PRJEB44214).

\section{Supplementary Information}

The online version contains supplementary material available at https://doi. org/10.1186/s13099-021-00477-7.

Additional file 1: Table S1. Heat map showing only bacterial taxa where significant changes in relative abundance were present in at least one of the pairwise comparisons.

Additional file 2: Figure S1. PCoA showing similarities/differences between bacterial communities from different colonic segments. In all pairwise comparisons between the four groups, only differences between ascending vs sigmoid colonic communities were statistically significant $\left(\right.$ PERMANOVA $P=0.0449, R^{2}=0.0855, F=1.4952$ )

\section{Acknowledgements}

The authors would like to thank Dr Ziad Abbassi, Dr Arnaud Dupuis, Dr Emilie Liot, Dr Naiken Surennaidoo (Division of Digestive Surgery, Geneva University Hospitals) for their help in recruiting patients and obtaining the samples, as well as Monica Perez, RN, Americo Agostinho, RN, and Catherine BandeiraClerc, RN (Infection Control Programme, Geneva University Hospitals) for conducting the SSI surveillance activities.

\section{Authors' contributions}

MA, SH, JS and VL designed the study. MA, NCB, VD, DOA, FR and JM recruited patients. MA, DOA, JM, VD were responsible for the sample management. MG performed wet lab work for NGS. NG performed the bioinformatics analyses. $M A$ and $V L$ wrote the first draft of the manuscript. NCB, VD, DOA, JM, FR and
$\mathrm{SH}$ provided major contributions to the manuscript. All authors read and approved the final manuscript.

\section{Funding}

This study was funded by the Research and Development Programme scheme (Grant No. PRD 21-2018-II) from Geneva University Hospitals.

\section{Declarations}

Ethics approval and consent to participate

The study was approved by the local Ethics Committee (Geneva, no. 201802379) and complied with the declaration of Helsinki. All participants provided written informed consent.

\section{Consent for publication}

Not applicable.

\section{Competing interests}

The authors declare that they have no competing interests.

\section{Author details}

${ }^{1}$ Infection Control Programme, Geneva University Hospitals and Faculty of Medicine, Geneva, Switzerland. ${ }^{2}$ Genomic Research Laboratory, Division of Infectious Diseases, University Hospitals and University of Geneva, Geneva, Switzerland. ${ }^{3}$ Division of Digestive Surgery, Geneva University Hospitals, Geneva, Switzerland. ${ }^{4}$ Division of Infectious Diseases, Geneva University Hospitals, Geneva, Switzerland. ${ }^{5}$ Laboratory of Bacteriology, Geneva University Hospitals, Geneva, Switzerland

Received: 28 September 2021 Accepted: 17 December 2021

Published online: 04 January 2022

\section{References}

1. Suetens C, Latour K, Karki T, Ricchizzi E, Kinross P, Moro ML, et al. Prevalence of healthcare-associated infections, estimated incidence and composite antimicrobial resistance index in acute care hospitals and long-term care facilities: results from two European point prevalence surveys, 2016 to 2017. Euro Surveill. 2018;23(46):1800516.

2. Meyer J, Naiken S, Christou N, Liot E, Toso C, Buchs NC, et al. Reducing anastomotic leak in colorectal surgery: the old dogmas and the new challenges. World J Gastroenterol. 2019;25(34):5017-25.

3. Troillet N, Aghayev E, Eisenring MC, Widmer A, Swissnoso. First results of the Swiss National Surgical Site Infection Surveillance Program: who seeks shall find. Infect Control Hosp Epidemiol. 2017;38:697-704.

4. Silvestri M, Dobrinja C, Scomersi S, Giudici F, Turoldo A, Princic E, et al. Modifiable and non-modifiable risk factors for surgical site infection after colorectal surgery: a single-center experience. Surg Today. 2017:48:338-45.

5. Gervaz P, Bandiera-Clerc C, Buchs NC, Eisenring MC, Troillet N, Perneger $\mathrm{T}$, et al. Scoring system to predict the risk of surgical-site infection after colorectal resection. Br J Surg. 2012;99(4):589-95.

6. Drosdeck J, Harzman A, Suzo A, Arnold M, Abdel-Rasoul M, Husain S. Multivariate analysis of risk factors for surgical site infection after laparoscopic colorectal surgery. Surg Endosc. 2013;27(12):4574-80.

7. Blumetti J, Luu M, Sarosi G, Hartless K, McFarlin J, Parker B, et al. Surgical site infections after colorectal surgery: do risk factors vary depending on the type of infection considered? Surgery. 2007;142(5):704-11.

8. Hourigan JS. Impact of obesity on surgical site infection in colon and rectal surgery. Clin Colon Rectal Surg. 2011;24(4):283-90.

9. Morikane K, Honda H, Yamagishi T, Suzuki S, Aminaka M. Factors associated with surgical site infection in colorectal surgery: the Japan nosocomial infections surveillance. Infect Control Hosp Epidemiol. 2014;35(6):660-6.

10. Pedroso-Fernandez Y, Aguirre-Jaime A, Ramos MJ, Hernandez M, Cuervo $\mathrm{M}$, Bravo A, et al. Prediction of surgical site infection after colorectal surgery. Am J Infect Control. 2016;44(4):450-4.

11. Grant R, Aupee M, Buchs NC, Cooper K, Eisenring MC, Lamagni T, et al. Performance of surgical site infection risk prediction models in colorectal 
surgery: external validity assessment from three European national surveillance networks. Infect Control Hosp Epidemiol. 2019;40(9):983-90.

12. Guyton K, Alverdy JC. The gut microbiota and gastrointestinal surgery. Nat Rev Gastroenterol Hepatol. 2017;14(1):43-54

13. Allegranzi B, Bischoff P, de Jonge S, Kubilay NZ, Zayed B, Gomes SM, et al. New WHO recommendations on preoperative measures for surgical site infection prevention: an evidence-based global perspective. Lancet Infect Dis. 2016;16(12):e276-87.

14. Dubinsky-Pertzov B, Temkin E, Harbarth S, Fankhauser-Rodriguez C, Carevic B, Radovanovic I, et al. Carriage of extended-spectrum betalactamase-producing Enterobacteriaceae and the risk of surgical site infection after colorectal surgery: a prospective cohort study. Clin Infect Dis. 2018;68:1699-704.

15. Ostermann S, Morel P, Chale JJ, Bucher P, Konrad B, Meier RPH, et al. Randomized controlled trial of enhanced recovery program dedicated to elderly patients after colorectal surgery. Dis Colon Rectum. 2019;62(9):1105-16.

16. Weimann A, Braga M, Carli F, Higashiguchi T, Hubner M, Klek S, et al. ESPEN guideline: clinical nutrition in surgery. Clin Nutr. 2017;36(3):623-50.

17. Somm E, Montandon SA, Loizides-Mangold U, Gaia N, Lazarevic V, De Vito C, et al. The GLP-1R agonist liraglutide limits hepatic lipotoxicity and inflammatory response in mice fed a methionine-choline deficient diet. Transl Res. 2021;227:75-88.

18. Zhang J, Kobert K, Flouri T, Stamatakis A. PEAR: a fast and accurate Illumina Paired-End reAd mergeR. Bioinformatics. 2014;30(5):614-20.

19. Edgar RC. UPARSE: highly accurate OTU sequences from microbial amplicon reads. Nat Methods. 2013;10(10):996-8.

20. Edgar RC. Search and clustering orders of magnitude faster than BLAST. Bioinformatics. 2010;26(19):2460-1.

21. Yoon S-H, Ha S-M, Kwon S, Lim J, Kim Y, Seo H, et al. Introducing EzBioCloud: a taxonomically united database of $16 \mathrm{~S}$ rRNA gene sequences and whole-genome assemblies. Int J Syst Evol Microbiol. 2017;67(5):1613-7.

22. Edgar RC. Search and clustering orders of magnitude faster than BLAST. Bioinformatics. 2010;26:2460-1.

23. Schloss PD, Westcott SL, Ryabin T, Hall JR, Hartmann M, Hollister EB, et al. Introducing mothur: open-source, platform-independent, communitysupported software for describing and comparing microbial communities. Appl Environ Microbiol. 2009;75(23):7537-41.

24. Bray R, Curtis JT. An ordination of the upland forest communities of southern Wisconsin. Ecol Monograph. 1957;27(4):325-34.

25. Anderson MJ. Permutational Multivariate Analysis of Variance (PERMANOVA). Wiley StatsRef: Statistics Reference Online2017. p. 1-15.

26. Anderson MJ. Distance-based tests for homogeneity of multivariate dispersions. Biometrics. 2006;62(1):245-53.

27. Oksanen J, Blanchet FG, Friendly M, Kindt R, Legendre P, McGlinn D, et al. Vegan: Community Ecology Package (Version 2.5-7). 2020. https://cran.rproject.org/web/packages/vegan/index.html.

28. Mandal S, Van Treuren W, White RA, Eggesbo M, Knight R, Peddada SD Analysis of composition of microbiomes: a novel method for studying microbial composition. Microb Ecol Health Dis. 2015;26:27663.

29. Donaldson GP, Lee SM, Mazmanian SK. Gut biogeography of the bacterial microbiota. Nat Rev Microbiol. 2016;14(1):20-32.

30. Shogan BD, Chen J, Duchalais E, Collins D, Chang M, Krull K, et al. Alterations of the rectal microbiome are associated with the development of postoperative ileus in patients undergoing colorectal surgery. J Gastrointest Surg. 2020;24(7):1663-72.

31. van Praagh JB, de Goffau MC, Bakker IS, van Goor H, Harmsen HJM, Olinga P, et al. Mucus microbiome of anastomotic tissue during surgery has predictive value for colorectal anastomotic leakage. Ann Surg. 2019;269(5):911-6.

32. van Praagh JB, de Goffau MC, Bakker IS, Harmsen HJ, Olinga P, Havenga K. Intestinal microbiota and anastomotic leakage of stapled colorectal anastomoses: a pilot study. Surg Endosc. 2016;30(6):2259-65.

33. Jin Y, Geng R, Liu Y, Liu L, Jin X, Zhao F, et al. Prediction of postoperative ileus in patients with colorectal cancer by preoperative gut microbiota. Front Oncol. 2020;10:526009.

34. Ohigashi S, Sudo K, Kobayashi D, Takahashi T, Nomoto K, Onodera H. Significant changes in the intestinal environment after surgery in patients with colorectal cancer. J Gastrointest Surg. 2013;17(9):1657-64.
35. Lederer AK, Pisarski P, Kousoulas L, Fichtner-Feigl S, Hess C, Huber R. Postoperative changes of the microbiome: are surgical complications related to the gut flora? A systematic review. BMC Surg. 2017;17(1):125.

36. Mizuta M, Endo I, Yamamoto S, Inokawa H, Kubo M, Udaka T, et al. Perioperative supplementation with bifidobacteria improves postoperative nutritional recovery, inflammatory response, and fecal microbiota in patients undergoing colorectal surgery: a prospective, randomized clinical trial. Biosci Microbiota Food Health. 2016;35(2):77-87.

37. Shogan BD, Smith DP, Christley S, Gilbert JA, Zaborina O, Alverdy JC. Intestinal anastomotic injury alters spatially defined microbiome composition and function. Microbiome. 2014;2:35.

38. Holder-Murray J, Yeh A, Rogers MB, Firek B, Mahler B, Medich D, et al. Time-dependent displacement of commensal skin microbes by pathogens at the site of colorectal surgery. Clin Infect Dis. 2020;73:e2754-62.

39. Wenzel RP. Surgical site infections and the microbiome: an updated perspective. Infect Control Hosp Epidemiol. 2019;40(5):590-6.

40. Agnes A, Puccioni C, D'Ugo D, Gasbarrini A, Biondi A, Persiani R. The gut microbiota and colorectal surgery outcomes: facts or hype? A narrative review. BMC Surg. 2021;21(1):83.

41. Veziant J, Poirot K, Chevarin C, Cassagnes L, Sauvanet P, Chassaing B, et al. Prognostic value of a combination of innovative factors (gut microbiota, sarcopenia, obesity, metabolic syndrome) to predict surgical/oncologic outcomes following surgery for sporadic colorectal cancer: a prospective cohort study protocol (METABIOTE). BMJ Open. 2020;10(1):e031472.

42. Zingg W, Park BJ, Storr J, Ahmad R, Tarrant C, Castro-Sanchez E, et al. Technology for the prevention of antimicrobial resistance and healthcareassociated infections; 2017 Geneva IPC-ThinkTank (Part 2). Antimicrob Resist Infect Control. 2019;8:83.

43. Bassis CM, Moore NM, Lolans K, Seekatz AM, Weinstein RA, Young VB, et al, Comparison of stool versus rectal swab samples and storage conditions on bacterial community profiles. BMC Microbiol. 2017;17(1):78.

44. Reyman M, van Houten MA, Arp K, Sanders EAM, Bogaert D. Rectal swabs are a reliable proxy for faecal samples in infant gut microbiota research based on 16S-rRNA sequencing. Sci Rep. 2019;9(1):16072.

\section{Publisher's Note}

Springer Nature remains neutral with regard to jurisdictional claims in published maps and institutional affiliations.

Ready to submit your research? Choose BMC and benefit from

- fast, convenient online submission

- thorough peer review by experienced researchers in your field

- rapid publication on acceptance

- support for research data, including large and complex data types

- gold Open Access which fosters wider collaboration and increased citations

- maximum visibility for your research: over 100M website views per year

At BMC, research is always in progress.

Learn more biomedcentral.com/submissions 